\title{
Análise do desempenho motor de escolares praticantes de futsal
} e voleibol

\author{
Analysis of motor performance of school practitioners of indoor soccer \\ and volleyball
}

\author{
R. Drews, P.L. Cardozo, S.T. Corazza, F.S. Flôres
}

ARTIGO ORIGINAL | ORIGINAL ARTICLE O objetivo do presente estudo foi analisar o desempenho motor de escolares a partir da prática regular de futsal e voleibol. A amostra foi constituida por 60 indivíduos do sexo masculino, com idade média de $16 \pm 0.4$ anos, divididos em dois grupos: praticantes de futsal $(\mathrm{GF})(n=32)$ e praticantes de voleibol $(\mathrm{GV})(n=28)$. Os indivíduos analisados praticavam regularmente 2 aulas semanais de 60 minutos em suas respectivas modalidades esportivas, no período mínimo de 6 meses. Para avaliar as capacidades físico-motoras foram utilizados os protocolos de teste Shuttle Run ou Corrida do Vai-eVem de Johnson e Nelson (1979) para agilidade, software de Pereira, Teixeira, Villis, e Corazza (2009) para o tempo de reação simples e escolha, e o cinesiômetro (Paixão, 1981) e goniômetro (Hurley, Rees, \& Newham, 1998) para propriocepção de membros superiores e inferiores respectivamente. Para a análise dos dados, fez-se uso da estatística inferencial, com o Teste $t$ para amostras independentes, através do pacote estatístico SPSS versão 14.0, com nível de significância alfa de 5\%. Os resultados não apresentaram diferença estatisticamente significativa entre os grupos em nenhuma capacidade físicomotora analisada.

Palavras-chave: agilidade, educação física e treinamento, homem, tempo de reação, propriocepção, voleibol

The aim of this study was to analyze the motor performance of students from the regular practice of indoor soccer and volleyball. The study group consisted of 60 males, mean age $16 \pm 0.4$ years, divided into two groups: indoor soccer $(\mathrm{GF})(n=32)$ and volleyball players $(\mathrm{GV})(n=28)$. All individuals analyzed possessed a regular practice of two weekly classes of 60 minutes each session in their respective sports, at least 6 months of practice and could not perform other physical activities/sports regularly in extracurricular period. To evaluate the physical and motor skills were used in testing protocols Shuttle Run or Race-and-Go Comes Johnson and Nelson (1979) for agility, software Pereira, Teixeira, Villis, and Corazza (2009) for the TRS and TRE, and cinesiômetro (Paixão, 1981) and goniometer (Hurley, Rees, \& Newham, 1998) proprioception to the upper and lower limbs respectively. For data analysis, was made use of descriptive statistics, with the $t$ test for independent samples, using the statistical package SPSS version 14.0, with a significance level alpha of $5 \%$. The results showed no statistically significant difference between groups in any physical-motor ability assessed.

Keywords: agility, physical education and training, man, reaction time, proprioception, volleyball

Submetido: 29.08.2012 | Aceite: 26.01.2013

Ricardo Drews. Universidade Federal de Pelotas- UFPel, Brasil.

Priscila Lopes Cardozo, Sara Teresinha Corazza, Fábio Saraiva Flôres. Universidade Federal de Santa Maria- UFSM, Brasil.

Endereço para correspondência: Ricardo Drews, Rua Almirante Tamandaré, 251, apt 302, CEP 96010750, Pelotas, RS, Brasil.

E-mail: ricardodrews@hotmail.com 
Um bom desempenho motor é um atributo fundamental no repertório motor de crianças e adolescentes, tornando-se essencial para a efetiva participação em diferentes programas de atividade física. Em função disso, tem crescido consideravelmente o número de estudos (Lopes, Maia, Silva, Seabra, \& Morais, 2003; Rebelo \& Oliveira, 2004; Vidal et al., 2003) que buscam obter informações relativas ao índice e desenvolvimento de capacidades físico-motoras como o equilíbrio, tempo de reação, coordenação, agilidade, flexibilidade, entre outras utilizadas em diferentes locais e tipos de prática.

Segundo Schmidt e Wrisberg (2001), o estudo das capacidades físico-motoras é um tópico de relevância no cenário do comportamento motor, referindo-se ao entendimento de um dos assuntos pertinentes às diferenças individuais. Essas são definadas como traços gerais, estáveis e duradouros, as quais podem fazer parte da estrutura de várias habilidades motoras e, da mesma forma, uma habilidade motora pode ter, na sua estrutura, o envolvimento de várias capacidades (por exemplo, Magill, 2000; Schimdt \& Lee, 2011).

A prática de atividade física tem sido indicada para promoção de saúde, melhora da qualidade de vida e está intimamente ligada ao desenvolvimento de capacidades físico-motoras (Silva et al., 2009; Strong et al., 2005). Diferentes práticas de atividade física, entre elas a esportiva, tem se mostrado relevante na aquisição de uma vida mais saudável e no aprimoramento de diferentes capacidades físico-motoras. Segundo Guedes e Guedes (1996), o estilo de vida começa a ser formado na infância, indicando que crianças com baixo nível de atividade motora, podem tornar-se adultos sedentários e, consequentemente, não apresentarem um bom índice de qualidade de vida.

Estudos relacionados à prática de atividade física em crianças e adolescentes ainda estão caracterizados pela inatividade física, embora a Educação Física Escolar e, logo, a prática esportiva inserida nela, sejam uma das poucas ativi- dades praticadas por esta população (Fortes; Azevedo; Kremer; Hallal, 2012). O futsal e o voleibol são dois exemplos de modalidades esportivas desenvolvidas na Educação Física Escolar, as quais apresentam grande variedade de experiências físico-motoras, culminando num alto grau de habilidade e de eficiências nos gestos específicos assim como no aprendizado no seu sentido e significado (Mutti, 2003).

Para a prática do futsal e do voleibol, diferentes capacidades físico-motoras servem como base para a obtenção de um melhor aprendizado e desempenho, apesar de vários elementos serem necessários para aquisição dessas habilidades. No presente estudo explorou-se o tempo de reação (TR), agilidade e a propriocepção.

O TR pode ser definido como a velocidade e eficácia referentes à tomada de decisão de um indivíduo, ou ainda como o lapso temporal entre a apresentação de um estímulo e o início de uma resposta motora. Além disso, o tempo de reação pode ser dividido em: tempo de reação simples (TRS), quando apenas um estímulo ocorrerá e o indivíduo deverá responder prontamente a ele; tempo de reação de escolha (TRE), quando dois ou mais estímulos aparecerão e o indivíduo deverá identificá-los, e, por fim, selecionar a resposta mais adequada dentre as diferentes possibilidades (por exemplo, Magill, 2000; Miyamoto \& Meira Jr., 2004; Vagheti, Roesler, \& Andradell, 2007).

Outra capacidade físico-motora relevante para o presente estudo é a agilidade, definida segundo Guedes e Guedes (1996), como a capacidade de realização de movimentos de curta duração em alta intensidade, com mudanças de direção e/ou mudanças no centro de gravidade do corpo, com aceleração e desaceleração. Essa capacidade pode mudar de direção sem perda de velocidade, força, equilíbrio ou controle do corpo. Finalmente, a propriocepção refere-se ao desenvolvimento do sistema cinestésico, sendo importante tanto na manutenção das capacidades físico-motoras necessárias às atividades da vida diária, quanto na performance de habi- 
lidades esportivas. A mesma fornece informações importantes sobre as posições das partes do corpo, assim como as posições corporais no ambiente, e sua relação com diferentes objetos (Haywood \& Getchell, 2004).

O desenvolvimento dessas capacidades está diretamente relacionado com um bom nível de rendimento em diferentes habilidades motoras específicas (Silva et al., 2009;Vagheti et al., 2007), entre elas o futsal e o voleibol destacados acima. O sucesso nessas habilidades está intimamente relacionado a diversos fatores, tais como a percepção de estímulos, interpretação, programação e execução das respostas em intervalos curtos de tempo nas diversas situações de jogo.

Sabendo da importância dessas capacidades no desenvolvimento de modalidades esportivas, a agilidade, propriocepção e o tempo de reação vêm sendo investigados por vários estudiosos (por exemplo, Barcelos, Morales, Maciel, Azevedo, \& Silva, 2009; Chagas, 2005; Corazza, Pereira, \& Villis, 2005; Maciel et al., 2009; Maia, Vágula, Souza, \& Pereira, 2007; Pereira et al., 2009; Vaghetti et al., 2007) tanto em situações formais, quanto informais, a fim de entender seu desenvolvimento e diferenças em diversas vivências motoras no dia a dia de crianças, jovens e adultos. Porém, são limitados estudos apresentando qual prática esportiva possibilita um melhor desenvolvimento dessas capacidades físico-motoras.

Além disso, o presente estudo destaque-se por mensurar essas capacidades a partir de práticas esportivas no contexto escolar, diferindo da maioria dos estudos realizados em clubes esportivos (Neto, Barbieri, Barbieri, \& Gobbi, 2009). Entretanto, deve ser levado em consideração que a prática esportiva na Educação Física Escolar, além de contribuir com o desenvolvimento de habilidades básicas e o aprimoramento de capacidades físico-motoras, objetiva também desenvolver a postura crítica dos alunos perante as atividades da cultura corporal, para a aquisição de autonomia de conhecimentos necessários a uma prática intencional e permanente
(Jeber, 1997; Vago, 1999).

Diante desses argumentos, o objetivo do presente estudo foi analisar o desempenho motor de escolares a partir da prática regular de futsal e voleibol.

\section{MÉTODO}

Esta pesquisa, segundo Thomas, Nelson, e Silverman (2007), é do tipo descritiva-observacional, pois descreve quantitativamente o comportamento de grupos a partir das observações das capacidades físico-motoras agilidade, propriocepção e TR.

\section{Amostra}

A amostra selecionada foi constituída de 60 sujeitos, todos do sexo masculino, com idade média de $16 \pm 0.4$ anos, escolares participantes das aulas de Educação Física de duas escolas situadas na de cidade Santa Maria- RS/BR. Os indivíduos foram divididos em dois grupos conforme a modalidade esportiva realizada nas aulas de Educação Física: grupo de futsal (GF) $(n=32)$ e o grupo de voleibol (GV) $(n=$ 28). Todos os sujeitos realizavam uma prática regular de duas aulas semanais com duração de 60 minutos em sua respectiva modalidade esportiva.

Antes da realização da coleta de dados, o projeto do estudo foi submetido ao Comitê de Ética em Pesquisa da Universidade Federal de Santa Maria (UFSM) e, posteriormente aprovado com o protocolo de número 0126.0.243.000-11. Todos os sujeitos foram voluntários, sendo sua participação concedida após assinatura do Termo de Consentimento Livre e Esclarecido pelos pais ou responsáveis.

Os sujeitos incluídos no estudo deveriam ter $75 \%$ de frequência regular nas aulas de Educação Física, e não apresentar nenhuma alteração visual, somatossensorial, auditiva, ou ferimentos que impedissem ou dificultassem a realização dos testes. Do mesmo modo, não poderiam realizar outras atividades físicas/ esportivas regulares em período extracurricular 
e praticar somente a habilidade motora específica analisada nas aulas de Educação Física. Também foi levado em conta o tempo de prática das mesmas, sendo de no mínimo de seis meses.

\section{Instrumentos}

Os instrumentos utilizados para a realização da coleta de dados foram: o software de Pereira et al. (2009) para mensuração do TRS e TRE, instalado em um computador portátil marca Sony com um processador Pentium Dual-Core $2.26 \mathrm{GHz}$. Esse software foi desenvolvido na ferramenta Borland Delphi 7 que utiliza a linguagem de programação object pascal e, com isso, avalia os tempos de reação a partir de um estímulo visual e a reação do movimento do membro superior dominante avaliando, assim, o tempo de reação simples e de escolha.

Para mensurar a agilidade foi utilizado o Teste do Shuttle Run ou Corrida do vai-e-vem de Johnson e Nelson (1979). O teste consta de duas linhas paralelas traçadas no solo, com distância de 9.14 metros entre elas. Dois blocos de madeira são colocados a $10 \mathrm{~cm}$ da linha externa e separados entre si por um espaço de $30 \mathrm{~cm}$. Ao sinal de um comando o avaliado corre, pega um dos blocos e leva para traz da linha inicial, em seguida corre e pega o outro. O cronômetro, no qual é mensurado o tempo de movimento, é parado após colocar o $2^{\circ}$ bloco atrás da linha inicial. São realizadas duas tentativas, com intervalo de 1 minuto entre as mesmas, sendo escolhida aquela com o tempo mais satisfatório.

Em relação ao nível de propriocepção de membros superiores (PMS) e inferiores (PMI) foram utilizados o Cinesiômetro do protocolo de Paixão (1981) e o Goniômetro (Hurley et al., 1998), respectivamente. O cinesiômetro é um instrumento composto por uma base de madeira, onde estão afixados os graus de $0^{\circ}$ a $180^{\circ}$ para determinação de ângulos. No seu eixo principal encontra-se um braço móvel, fixado na parte central e sua extremidade podendo ser deslocada ao ponto inicial correspondente a $0^{\circ}$ e posteriormente a ângulos pré-determinados: $90^{\circ}$ para a direita; $45^{\circ}$ para a esquerda e, a partir do ponto de $45^{\circ}$, considerando-o como ponto $0^{\circ}$, movê-lo $60^{\circ}$ para a direita. À medida que se move o braço do instrumento, juntamente com o braço do sujeito, notifica-se a posição em que se está sem comunicar ao sujeito o ângulo correspondente. Após, volta-se à posição inicial $\left(0^{\circ}\right)$ e solicita-se ao sujeito que repita, na mesma ordem. Posteriormente são registrados os erros, em relação aos graus que faltam ou excedem o ângulo determinado.

Já o Goniômetro mensurou o ângulo da movimentação articular (extensão e flexão), no qual é fixado no membro inferior do voluntário por duas faixas de velcro, sendo conectadas na parte distal da coxa e a outra na parte proximal da perna. No momento da avaliação, o participante permaneceu sentado sobre uma mesa à altura de $1,20 \mathrm{~m}$, ajustado na articulação do joelho. Para remover informações visuais, os olhos foram vendados. Partindo-se de $90^{\circ}$ de flexão, a perna do avaliado foi movida passivamente em extensão até chegar à angulação que foi determinada por sorteio $\left(40^{\circ}\right)$. O membro foi mantido por dez segundos na mesma posição, e após retornando à posição inicial. Após cinco segundos, o participante foi instruído a efetuar ativamente o mesmo movimento, parando assim que percebesse que a posição alvo fosse atingida.

Posteriormente, o segundo teste foi aplicado, dessa vez partindo-se de $0^{\circ}$, flexionando o joelho até o ângulo estabelecido $\left(20^{\circ}\right)$, seguindo os mesmos critérios acima. Ressalta-se que tanto para cinesiômetro como goniômetro, foi utilizada a avaliação através do erro absoluto, ou seja, a diferença em graus entre o ângulo proposto e o ângulo reproduzido pelos sujeitos. Portanto, quanto menor o erro, melhor será o nível de propriocepção.

\section{Procedimentos}

Primeiramente, foi feito contato com as escolas para explicar o objetivo do estudo e 
verificar a disponibilidade de tempo e espaço. Após o consentimento da direção da escola, e a devolução do Termo de Consentimento Livre e Esclarecido com as respectivas assinaturas de pais ou responsáveis, deu-se início ao processo de coleta dos dados.

Cada um dos 60 participantes realizou os cinco testes na seguinte ordem: TRS, TRE, PMS, PMI e agilidade. O intervalo entre os testes foi de 10 minutos, não sendo informados os resultados ou qualquer outra forma de feedback durante e após a prática.

Todos os testes foram realizados nas dependências da escola, em um ambiente silencioso e por avaliadores treinados, antes do início das aulas de Educação Física para que não houvesse nenhum tipo de alteração nos testes. Durante o processo de coleta dos dados estiveram presente apenas o pesquisador e o sujeito, evitando assim interferências, distrações e eventuais constrangimentos.

\section{Análise Estatística}

Os dados das avaliações de agilidade, TRS, TRE, PMS e PMI, considerando tanto o grupo GV quanto GF, foram analisados quanto à distribuição utilizando-se da estatística $\mathrm{W}$ de Shapiro-Wilk que revelou distribuição normal $(p>.05)$.

Para responder as questões do estudo, utilizou-se a estatística descritiva com médias, desvios-padrão, e o mínimo e o máximo escore; como estatística de inferência utilizou-se o Teste t Student para amostras independentes, para verificar a diferença entre os grupos (futsal $\mathrm{X}$ voleibol). Para a realização dos procedimentos estatísticos foi utilizado o pacote estatístico SPSS for Windows 14.0, com um nível de significância alfa de $5 \%$.

\section{RESULTADOS}

As informações sobre os resultados obtidos nos testes de agilidade, TRS, TRE, PMS e PMI, demonstrados através de médias, desvios-padrão e dos escores mínimos e máximos, e as análises de diferença intergrupos (GF x GV) das variáveis analisadas (agilidade, TRS, TRE, PMS, PMI) são apresentados na Tabela 1.

A partir das observações realizadas, nenhuma interação entre os grupos apresentou diferença estatisticamente significativa $(p>$ .05). É importante destacar que, em termos de desempenho, em relação à agilidade, TRS e TRE, quanto menor o escore apresentado, melhor o desempenho nos seus respectivos testes. No caso da propriocepção, os valores mais próximos a $40^{\circ}$ e $20^{\circ}$ graus, apresentam melhor performance de PMS e PMI respectivamente.

\section{DISCUSSÃO}

O presente estudo analisou o desempenho

Tabela 1

Análise das variáveis agilidade, tempo de reação simples (TRS), tempo de reação de escolha (TRE), propriocepção de membros superiores (PMS) e propriocepção de membros inferiores (PMI) intergrupos

\begin{tabular}{|c|c|c|c|c|c|c|c|}
\hline Variáveis & $\mathrm{N}$ & Mínimo & Máximo & Média & Desvio Padrão & $t$ & $p$ \\
\hline Agilidade* (s) & 32 & 8.9 & 11.7 & 9.9 & .7 & \multirow{2}{*}{-1.17} & \multirow{2}{*}{.25} \\
\hline Agilidade $^{* *}(\mathrm{~s})$ & 28 & 8.4 & 12.3 & 9.7 & .8 & & \\
\hline TRS* (ms) & 32 & 275.1 & 569.0 & 372.5 & 80.6 & \multirow{2}{*}{.53} & \multirow{2}{*}{.59} \\
\hline $\mathrm{TRS}^{* *}(\mathrm{~ms})$ & 28 & 247.3 & 821.1 & 368.8 & 119.6 & & \\
\hline $\mathrm{TRE}^{* *}$ (ms) & 32 & 402.9 & 897.0 & 567.8 & 118.2 & \multirow{2}{*}{.43} & \multirow{2}{*}{.67} \\
\hline $\mathrm{TRE}^{* *}$ (ms) & 28 & 396.3 & 864.4 & 517.0 & 138.2 & & \\
\hline PMS* $\left.{ }^{\circ}\right)$ & 32 & 7.0 & 57.0 & 26.1 & 14.3 & \multirow{2}{*}{.88} & \multirow{2}{*}{.38} \\
\hline $\mathrm{PMS}^{* *}\left({ }^{\circ}\right)$ & 28 & 2.0 & 66.0 & 23.0 & 15.1 & & \\
\hline $\mathrm{PMI}^{*}\left({ }^{\circ}\right)$ & 32 & 2.0 & 32.0 & 14.4 & 6.8 & \multirow{2}{*}{.08} & \multirow{2}{*}{.93} \\
\hline $\mathrm{PMI}^{* *}\left({ }^{\circ}\right)$ & 28 & 2.0 & 29.0 & 14.3 & 8.1 & & \\
\hline
\end{tabular}

Nota: ${ }^{*}=$ grupo de praticantes de futsal; ${ }^{* *}=$ grupo de praticantes de voleibol; $\mathrm{s}=$ segundos; $\mathrm{ms}=$ milésimos de segundos; ${ }^{\circ}=$ graus 
motor de escolares a partir da prática regular de futsal e voleibol. Os resultados encontrados não apresentaram diferença estatisticamente significativa intergrupos para as variáveis analisadas (agilidade, TRS, TRE,PMI e PMS). Entretanto, uma análise mais específica dos resultados relacionando-os a outros estudos com as mesmas variáveis, sugere que os resultados deste estudo teriam sofrido a influência de alguns fatores essenciais no desenvolvimento de diferentes capacidades físico-motoras, desencadeado em resultados, decerto modo, esperados pela maneira que as diferentes habilidades motoras específicas são desenvolvidas em seus respectivos grupos.

O refinamento dessas habilidades motoras específicas como, o futsal e o voleibol sofre grande influência do nível das capacidades físico-motoras. Para que o indivíduo tenha total controle sobre as técnicas individuais de um desporto, é preciso que ele tenha controle sobre suas ações motoras (Ferreira, 1998).

Uma possível justificativa para os achados do presente estudo seriam a intensidade da prática e o objetivo das aulas da Educação Física na qual são trabalhadas as duas habilidades motoras específicas analisadas. Nesse contexto, a Educação Física Escolar, entre um dos seus objetivos, busca introduzir e integrar o aluno na cultura corporal de movimento, formando o cidadão que vai produzí-la, reproduzí-la e transformá-la, instrumentalizando-o para usufruir do jogo, do esporte, das atividades rítmicas e dança, das ginásticas e práticas de aptidão física, em benefício da qualidade da vida (Betti, 1992). Ao contrário dos objetivos de treinamento de equipes e atletas de alto rendimento, que visam atingir um alto nível de desempenho em dada circunstância, baseado em um conceito de periodização dividido em fases e princípios de treinamento (Bompa, 2001).

Observando o TRS e TRE, que apesar de não apresentar diferença estatisticamente significativa $(p>.05)$ na comparação entre os grupos, não se pode ignorar que estas variáveis são de suma importância no aprendizado de diversas habilidades motoras específicas . No futsal e no voleibol, um melhor aprimoramento do tempo de reação pode proporcionar a capacidade de responder prontamente a um estímulo, sendo vital para o sucesso de diversos gestos motores no jogo em si (Barcelos et al., 2009; Chagas, 2005).

Em um estudo analisando o TRS, Vaghetti et al. (2007) buscaram verificar diferenças no TRS em adultos profissionais, amadores e praticantes de surf. Os resultados mostraram uma média do TRS para os profissionais de 213.0 \pm 59 milésimos de segundos, nos amadores de $218.0 \pm 80.6$ milésimos de segundos e para os praticantes da modalidade $289.0 \pm 98$ milésimos de segundos. Assim, demonstrando uma grande diferença nos resultados dos atletas profissionais e amadores em relação aos indivíduos que apenas praticavam a modalidade. Este fato pode ser comparado aos resultados de TRS do presente estudo, no qual foram encontrados $372.5 \pm 80.6$ milésimos de segundos para os praticantes da modalidade de futsal e $368.8 \pm$ 119.2 milésimos de segundos para praticantes da modalidade de voleibol, denotando que a prática realizada por atletas traz diferenças no desempenho quando comparados a escolares.

Esse achado proporciona uma relação ao modo e aos objetivos das habilidades motoras específicas trabalhadas dentro das aulas de Educação Física, em que segundo os PCN's (1997), é uma disciplina escolar que deve tratar da cultura corporal, com a finalidade de introduzir e integrar o aluno, formando um cidadão que vá produzir, reproduzir e também transformar, usufruindo de jogos, esportes, danças, lutas e ginásticas, em benefício do exercício crítico da cidadania e da melhoria da qualidade de vida. Em consequência disso, são desenvolvidas aulas de qualidade, porém o desenvolvimento motor e a sublimidade de algumas capacidades físico-motoras são negligenciadas. Diferentemente de clubes esportivos, onde 
há grande foco em relação ao aprimoramento dessas capacidades, proporcionando um melhor desempenho em outras ações motoras.

Pereira et al. (2009) também analisando o TRS e o TRE, investigaram a relação dessas variáveis no desempenho motor do nado crawl de sujeitos em diferentes estágios de aprendizagem, no qual foram utilizados os mesmos instrumentos de avaliação do TRS e TRE do presente estudo. Os resultados apontaram que o tempo de reação possui uma importância geral para o desempenho do nado, apresentando uma relevância maior no grupo dos avançados em relação aos intermediários e iniciantes. Houve uma grande diferença nas médias do TRS dos avançados (238.5 \pm 30 milésimos de segundos) e iniciantes (289.5 \pm 61 milésimos de segundos), como para o TRE dos avançados $(423.6 \pm 65$ milésimos de segundos) e iniciantes $(596.2 \pm 14$ milésimos de segundos). Comparando as médias entre os iniciantes e os dois grupos analisados no presente estudo, pode-se notar que apesar de valores e contextos diferentes, os dados apresentados podem ter sido influênciados pela intensidade de prática realizada.

Uma intensidade maior de prática pode proporcionar melhores resultados em relação a sujeitos com pouca prática e sem elementos motores mais apurados. Autores como Magill (2000) e Schmidt e Wrisberg (2001) acreditam que sujeitos com uma maior vivência e intensidade em uma modalidade esportiva, desenvolvam um maior potencial em algumas ações motoras. Isto porque, à medida que o sujeito se adapta à instabilidade natural do ambiente e à multiplicidade dos seus estímulos, a sua "capacidade cognitiva" cria a própria inteligência esportiva, podendo o mesmo solucionar de maneira mais rápida e correta situações inesperadas durante o jogo (Fontani, Maffei, Cameli, \& Polidori, 1999).

Neste contexto, é válido ressaltar que a vantagem de possuir uma boa capacidade de reação simples é uma possível redução do TRE, uma vez que o TRS é fortemente influenciado por aspectos genéticos como, por exemplo, a capacidade de condução neural (McMorris \& Keen, 1994). O TRE, além de apresentar esta mesma limitação em comum, ainda é determinado por aspectos de aprendizagem sendo, portanto, altamente influenciado pela prática (Schmidt \& Wrisberg, 2001).

Outro ponto a ser lembrado é que apesar de não ter sido encontrado uma diferença estatística entre os grupos, houve uma superioridade dos praticantes de voleibol em relação ao grupo de futsal tanto no TRS quanto no TRE. Apesar das duas habilidades motoras específicas necessitarem de um bom desenvolvimento dessas capacidades, a perspicácia de responder prontamente a um estímulo é importante para o sucesso de um atleta do voleibol. Para uma ação motora apropriada é necessário que o atleta detecte a ação do oponente para, então, tentar neutralizá-la (Volchan et al., 2003). A velocidade com que o atleta identifica a intenção do seu adversário está diretamente relacionada a um possível sucesso na resposta efetuada (Weineck, 2003). Neste contexto, ressalta-se a importância do tempo de reação.

Um estudo que demonstra a eficácia do TR foi de Maciel et al. (2009), que buscaram verificar a diferença de TRS em atletas de diferentes posições no voleibol. Os resultados apresentaram diferenças estatísticas em algumas posições, como nos ponteiros onde foi encontrada uma média de TRS de 271.1 \pm 55 milésimos de segundos, e opostos com uma média de 285.4 \pm 82 milésimos de segundos, comprovando a importância do TRS no voleibol atual. Dessa forma, foi verificado que os atletas que atuam como atacantes centrais apresentam maior velocidade de reação, seguido dos atacantes ponteiros, o que é coerente com as solicitações de movimento características dessas funções.

Outra variável analisada no presente estudo foi a agilidade. $\mathrm{Na}$ análise dos escores do teste de agilidade, também não foi encontrada diferença significativa entre os participantes dos 
dois grupos, porém esta capacidade também possui grande importância no aperfeiçoamento dessas habilidades motoras específicas. Segundo Cunha (2003), está capacidade desenvolve-se por meio de exercícios que exigem uma rápida inversão dos movimentos com participação de todo o corpo, e para os praticantes de voleibol e futsal, o desenvolvimento eficaz dessa capacidade é fundamental para melhorar os níveis de diversas ações motoras.

Como foi enfatizado nos resultados do TRS e TRE, a agilidade também pode ser afetada pelos objetivos presentes na Educação Física Escolar e a sua intensidade da prática. Um estudo que confirma as influências da intensidade de prática é de Neto et al. (2009). Os autores verificaram o efeito da prática sistemática de futebol no desempenho da agilidade, velocidade e coordenação de meninos de 10 e 11 anos, sendo o instrumento para medir a agilidade o mesmo utilizado no presente estudo. Participaram deste estudo crianças do sexo masculino, distribuídos em dois grupos: praticantes regulares e não-praticantes de futebol. Os resultados desse estudo demonstram superioridade dos sujeitos que possuem uma maior intensidade de prática, com um melhor desempenho da agilidade no grupo de praticantes de futebol. Esses achados revelam que através de programas de treinamento com o foco no alto rendimento há um melhor desenvolvimento de algumas capacidades físico-motoras. Diferentemente do que foi encontrado no presente estudo, no qual os indivíduos possuíam somente duas horas semanais de prática, sendo considerado uma baixa quantidade e intensidade para uma melhor performance de capacidades consideradas essenciais para algumas modalidades esportivas (Weineck, 2003).

Um estudo com características semelhantes com o presente estudo utilizando o mesmo instrumento, e que corrobora com os seus achados é de Silva et al. (2009). Foram mensuradas capacidades físico-motoras em escolares, entre elas a agilidade. A amostra foi composta por escolares com 13 anos de idade, submetidos a 16 semanas de prática regular de Educação Física. Os sujeitos foram divididos em dois grupos: no primeiro foi trabalhada a formação esportiva tradicional nas aulas de Educação Física (11.13 \pm 96 segundos); e o outro somente aulas teóricas de Educação Física (11.16 \pm 78 segundos). Não houve diferença estatística entre os dois grupos. Isso ressalta como a intensidade de prática pode influenciar o desempenho das capacidades, exigindo mudanças ou adaptações em atividades específicas que atendam as necessidades para um bom desempenho.

Em relação às variáveis PMS e PMI, também não foram encontradas diferenças estatisticamente significativas entre os grupos, o que também pode ser justificado pelos fatores citados nas outras variáveis analisadas. Há um número escasso de estudos que visam analisar o comportamento desta variável no desempenho motor de jovens. E, por sua vez, dificulta um melhor entendimento desta capacidade físico-motora em diferentes habilidades.

Antes, Contreira, Katzer, e Corazza (2009), utilizando o mesmo instrumento do presente estudo para avaliar a PMI, verificaram o nível proprioceptivo de membros inferiores de jovens e idosas praticantes de atividades físicas. Os resultados não apresentaram diferenças estatiscamente significativa entre os grupos, sendo encontrada uma média de erro absoluto nas idosas de $6.75 \pm 3.01^{\circ}$ e $5.73 \pm 4.24^{\circ}$ para jovens. Em comparação as médias verificadas no presente estudo, observou-se melhor desempenho tanto das idosas como das jovens em relação aos indivíduos praticantes de futsal e voleibol. Dessa forma, salienta-se a necessidade de um aprimoramento dessas capacidades nas atividades propostas nas aulas de Educação Física, para que os indivíduos obtenham um melhor desempenho na execução de diversas habilidades motoras específicas.

No estudo de Corazza, Pereira, e Villis (2005), ao analisar a propriocepção dos 
membros superiores de 8 mulheres praticantes da habilidade motora específica de natação no processo de familiarização ao meio líquido, também não foi encontrada diferença estatisticamente significativa entre os grupos, corroborando com os resultados do presente estudo. $\mathrm{O}$ erro médio dos indivíduos foi de $14.33 \pm 9.35^{\circ}$ no pré-teste e $6.58 \pm 5.44^{\circ}$ no pós-teste. $\mathrm{O}$ presente estudo apresentou médias inferiores de PMS em relação ao estudo citado, denotando o baixo nível de propriocepção dos sujeitos aqui analisados. Resultados semelhantes foram encontrados no estudo de Antes, Katzer e Corazza (2008), no qual verificaram a propriocepção de membros superiores de idosas praticantes de hidroginástica.

Uma das limitações do presente estudo foi não inserir um grupo controle, assim, dificultando a comparação com indivíduos que não praticam modalidades esportivas. Além disso, os testes motores utilizados neste estudo, aplicados em praticantes de futsal e voleibol, não foram desenvolvidos especificamente para estas modalidades, o que, de certa forma, limita uma análise mais consistente das informações sobre o desempenho das capacidades físico-motoras. Assim, sugere-se o desenvolvimento e validação de novos testes motores, sendo de níveis mais criteriosos e que atendam as especificidades do futsal e voleibol.

Pode-se ressaltar neste estudo, além dos resultados encontrados e suas relações com outras pesquisas, um elemento que é importante dentro da pedagogia do movimento e da aprendizagem motora, que é a forma como as atividades das referidas modalidades foram distribuídas. Nos grupos de estudo, os dois períodos de aula eram consecutivos, no mesmo dia. Tem-se pressupostos de Ellis (1977) e de Magill (2000) que abordam que a utilização otimizada do tempo de prática é crítica para aprendizagem efetiva, no sentido de que o mau uso do tempo disponível pode levar a uma quantidade de aprendizagem inferior à que teria ocorrido se o tempo de prática tivesse sido bem empregado. Então, se nestes grupos houvesse a substituição da prática caracterizada por massificada por uma distribuída, o desempenho motor nas capacidades agilidade, propriocepção e TR poderiam ser melhores, podendo ser apresentada uma superioridade de uma modalidade sobre a outra.

\section{CONCLUSÕES}

O presente estudo buscou verificar o efeito da prática regular de futsal e voleibol em aulas de Educação Física no desempenho motor de escolares. A partir dos resultados encontrados, conclui-se que apesar de não verificadas diferenças estatisticamente significativas das capacidades físico-motoras entre os grupos avaliados, essas capacidades são de suma importância para um melhor desenvolvimento de variadas habilidades motoras específicas como o futsal e o voleibol aqui analisados. Entretanto, muitos fatores podem ter acarretado esses resultados, como o objetivo da prática as quais foram submetidos e a sua intensidade, observando carências no desenvolvimento dessas capacidades dentro de cada modalidade esportiva. Com isso, percebe-se que professores de Educação Física como também técnicos esportivos devem atentar de forma importante para o desenvolvimento das capacidades físico-motoras e não somente da habilidade motora específica em si. Devido à importância da mensuração e aprimoramento do tempo de reação, agilidade e propriocepção, e de suas relações para um desempenho satisfatório no voleibol e futsal nas mais variadas experiências motoras, a presente avaliação pode favorecer o entendimento de algumas variáveis do processo de comportamento motor, permitindo que os profissionais envolvidos com a Educação Física consigam avaliar e intervir neste, por meio da adequação das atividades. Assim, sugere-se que novos estudos sejam conduzidos de forma a avaliar a qualidade das atividades motoras propostas em ambiente escolar como no caso das modalidades espor- 
tivas, e seu relacionamento com as capacidades físico-motoras, contribuindo para relação direta entre o tempo de reação, agilidade e propriocepção e sua melhor aplicabilidade em outras habilidades motoras presentes em diferentes contextos ou ambientes, buscando encontrar indicativos mais consistentes que ajudem a elucidar aspectos importantes para o entendimento destas variáveis.

\section{Agradecimentos:}

Nada declarado.

\section{Conflito de Interesses:}

Nada declarado.

\section{Financiamento:}

Nada declarado.

\section{REFERÊNCIAS}

Antes, D. L., Katzer, J. I., \& Corazza, S. T. (2008). Coordenação motora fina e propriocepção de idosas praticantes de hidroginástica. Revista Brasileira de Ciências do Envelhecimento Humano, 5(2), 24-32.

Antes, D. L., Contreira, A. R., Katzer, J. I., \& Corazza, S. T. (2009). Propriocepção de joelho em jovens e idosas praticantes de exercícios físicos. Fisioterapia e Pesquisa, 16(4), 306-310.

Barcelos, J. L., Morales, A. P., Maciel, R. N., Azevedo, M. M. A., \& Silva, V. F. (2009). Tempo de prática: estudo comparativo do tempo de reação motriz entre jogadoras de voleibol. Fitness Performance Journal, 8(2), 103-9.

Betti, M. (1992). Ensino de $1^{\circ}$. e $2^{\circ}$. graus: Educação Física para quê? Revista Brasileira de Ciências do Esporte, 13( 2), 282-7. doi: 10.1590/S010132892011000400001

Bompa, T. O. (2001). Treinamento de potência para o esporte. São Paulo: Phorte.

BRASIL. Ministério da Educação e Cultura. (1997). Parâmetros Curriculares Nacionais. Brasília: Secretaria de Educação Fundamental.

Bouchard, C., Dionne, F. T., Simeneau J., \& Boulay, M. R. (1992). Genetics of aerobic and anaerobic performances. Exercise and Sport Sciences Reviews. 20, 27-58.

Braz, T.V., Ugrinowitsch, H., Flausino, N.H., Freitas, W.Z., \& Piza, E.S. (2007). O Efeito da motivação no desempenho do teste de cooper em jovens futebolistas. Revista Treino e Desporto. $8(1), 53-9$.

Chagas, M. A. (2005). Associação entre Tempo de Reação e de movimento em jogadores de futsal. Revista Brasileira de Educação Física e Esporte, 19(4), 269-75.

Corazza, S. T., Pereira, E, F., \& Villis, J. M. C. (2005). Propriocepção e Familiarização ao meio líquido. Efdeportes.com - Revista Digital, 10(82). Consultado em 8 de fevereiro de 2012 a partir de http://www.efdeportes.com/efd82/propio.html

Cunha, F. A. (2003). Treinamento de velocidade e agilidade no futebol. Dicionário de Educação física e Esportes (2 ${ }^{\mathrm{a}}$ ed.). Barueri: Manole.

Ellis, H. C. (1977). Fundamentals of human learning, memory and cognition ( $2^{\mathrm{a}}$ ed.). Dubuque, Iowa: Wm. C. Brown Company Publishers.

Ferreira, N. S. C. (1998). Gestão democrática da Educação: atuais tendências, novos desafios. São Paulo: Cortez.

Fontani, G., Maffei, D., Cameli, S., \& Polidori, F. (1999). Reactivity and event-related potentials during attentional tests in athletes. European Journal of Applied Physiology, 80, 308-17. doi:10.1007/s00421-011-2246-z

Guedes, D. P., \& Guedes, J. E. R. P. (1996). Associação entre variáveis do aspecto morfológico e desempenho motor em crianças e adolescentes. Revista Paulista de Educação Física, 2(10), 99-112.

Haywood, K., \& Getchell. N. (2004). Desenvolvimento Motor ao longo da vida ( $3^{\mathrm{a}}$ ed.). Porto Alegre: Artmed.

Hurley, M.V., Rees, J., \& Newham, D.J. (1998). Quadriceps function, proprioceptive acuity and functional performance in healthy young, middle-aged and elderly subjects. Age Ageing, $27(1), 55-62$.

Johnson, B. L., \& Nelson, J. K. (1979). Pratical Measurements for Evaluation in Physical Education. United States of America: Burgess Publishing. 
Jeber, L. J. (1997). Plano de ensino em Educação Física escolar: um projeto político pedagógico em ação. In: Trilhas e partilhas: Educação Física na cultura escolar e nas práticas sociais (pp.113-143). Belo Horizonte: Cultura.

Lopes, V. P., Maia, J. A. R., Silva, R. G., Seabra, A., \& Morais, F. P. (2003). Estudo do nível de desenvolvimento da coordenação motora da população escolar (6 a 10 anos de idade) da Região Autónoma dos Açores. Revista Portuguesa de Ciências do Desporto, 3(1), 47-60.

Maciel, R. N., Morales, A. P., Barcelos, J. L., Nunes, W. J., Azevedo, M. M. A., \& Silva, V. F. (2009). Relação entre tempo de reação e função específica em jogadores de voleibol. Fitness Performance Journal, 8(6), 395-9.

Magill, R. A. (2000). Aprendizagem motora: conceitos e aplicações ( $5^{\mathrm{a}}$ ed.). São Paulo: Edgard Blücher.

Maia, M. A. C., Vágula, S., Souza, V. F. M., \& Pereira, V. R. (2007). Estudo comparativo da agilidade entre praticantes de dança de salão. Sabios: Revista Saúde e Biologia, 2(2), 37-44.

Mcmorris, T., \& Keen, P. (1994). Effect of exercise on simple reaction times of recreational athletes. Perceptual and Motor Skills, Missoula, 78, 123-30. doi: 10.2466/PMS.112.3.i-xii

Mutti, D. (2003). Futsal da iniciação ao alto nível (2a ed.). São Paulo: Phorte.

Miyamoto, R. J., \& Meira Jr., C. M.(2004). Tempo de reação e tempo das provas de 50 e 100 metros rasos do atletismo em federados e não federados. Revista Portuguesa de Ciências do Desporto, 4(3), 42-48.

Neto, O. B., Barbieri, F. A., Barbieri, R. A., \& Gobbi, L. T. B. (2009). Desempenho da agilidade, velocidade e coordenação de meninos praticantes e não praticantes de futebol. Fitness Performance Journal, 8(2), 110-4.

Paixão, J. S. (1981). Efeitos do Plano na Aquisição, Retenção e Transferência de uma Destreza Fechada - Dissertação de Mestrado não-publicada, Universidade Federal de Santa Maria, Santa Maria, Brasil.

Pereira, E. F., Teixeira, C. S., Villis, J. M. C., \& Corazza, S. T. (2009). Tempo de reação e desem- penho motor do nado crawl em diferentes estágios de aprendizagem. Revista Fisioterapia e Movimento, $22(4)$, 585-594.

Rebelo, A. N., \& Oliveira, J. (2004). Relação entre a velocidade, a agilidade e a potência muscular de futebolistas profissionais. Revista Portuguesa de Ciências do Desporto, 4(3), 342-348.

Schmitd, R. A., \& Wrisberg, C. A. (2001). Aprendizagem e performance motora: uma abordagem da aprendizagem baseada no problema ( $2^{\mathrm{a}}$ ed.). Porto Alegre: Artmed.

Schmidt, R., \& Lee, T. (2011). Motor control and learning: A behavioral emphasis ( $5^{\mathrm{a}} \mathrm{ed}$.). Champaign, IL: Human Kinetics Publishers.

Silva, J. L. B., Saraiva, A. R., Monte Júnior G. C., Portal, M. N. D., Lima, J. R. P., \& Dantas, E. H. M. (2009). Qualidades físicas de escolares de 13 anos submetidos à formação esportiva tradicional. Fitness Performance Journal, 8(6), 400-6.

Strong, W.B., Malina, R.M., Blimkie, C.J.R., Daniels, S.R., Dishman, R.K., Albert, B.G.C.,...Trudeau, F. (2005). Evidence Based Physical Activity For School-Age Youth. The Journal Of Pediatrics, 146, 732-737. doi: 10.1016/j.jpeds.2012.07.021

Thomas, J.R., Nelson, J.K., \& Silverman, S.J. (2007). Métodos de pesquisa em atividade física ( $5^{\mathrm{a}}$ ed.). Porto Alegre, RS: Artmed.

Vagheti, C. A. O., Roesler, H., \& Andradell, A. (2007). Tempo de reação simples auditivo e visual em surfistas com diferentes níveis de habilidade: comparação entre atletas profissionais, amadores e praticantes. Revista Brasileira de Medicina do Esporte, 13(2), 81-85. doi: 10.1590/ S1517-86922010000300007

Vago, T. M. (1999). Início e fim do século XX: maneiras de fazer educação física na escola. Cadernos Cedes, Corpo e educação. Campinas, Ano XIX, 48, 30-51. doi: 10.1590/S010132622001000100003

Vidal, S. M., Bustamante, A., Lopes, V. T., Seabra, A., Silva, R. G., \& Maia, J. A. (2003). Construção de cartas centílicas da coordenação motora de crianças dos 6 aos 11 anos da Região Autónoma dos Açores. Revista Portuguesa de Ciências do Desporto, 9(1), 24-35. 
116 | R. Drews, P.L. Cardozo, S.T. Corazza, F.S. Flôres

Volchan, E., Mirtes, M. G., Oliveira, L., Vargas, C., Mourão-Miranda, J., \& Azevedo, T. M. (2003).

Weineck, J. (2003). Treinamento ideal. São Paulo: Estímulos emocionais: processamento sensoManole.

rial e respostas motoras. Revista Brasileira de

Psiquiatria, 25, 29-32. doi: 10.1590/S1516-

44462003000600007

(c) EY-No Todo o conteúdo da revista Motricidade está licenciado sob a Creative Commons, exceto quando especificado em contrário e nos conteúdos retirados de outras fontes bibliográficas. 